\title{
Using the Multiphase Optimization Strategy (MOST) framework to test intervention delivery strategies: a study protocol
}

Sarabeth Broder-Fingert ${ }^{1,2^{*}}$, Jocelyn Kuhn ${ }^{1}$, Radley Christopher Sheldrick ${ }^{3}$, Andrea Chu ${ }^{1,3}$, Lisa Fortuna ${ }^{1,2}$, Megan Jordan ${ }^{4}$, Dana Rubin ${ }^{2,4}$ and Emily Feinberg ${ }^{1,2,3,4}$

\begin{abstract}
Background: Delivery of behavioral interventions is complex, as the majority of interventions consist of multiple components used either simultaneously, sequentially, or both. The importance of clearly delineating delivery strategies within these complex interventions-and furthermore understanding the impact of each strategy on effectiveness - has recently emerged as an important facet of intervention research. Yet, few methodologies exist to prospectively test the effectiveness of delivery strategies and how they impact implementation. In the current paper, we describe a study protocol for a large randomized controlled trial in which we will use the Multiphase Optimization Strategy (MOST), a novel framework developed to optimize interventions, i.e., to test the effectiveness of intervention delivery strategies using a factorial design. We apply this framework to delivery of Family Navigation (FN), an evidence-based care management strategy designed to reduce disparities and improve access to behavioral health services, and test four components related to its implementation.

Methods/design: The MOST framework contains three distinct phases: Preparation, Optimization, and Evaluation. The Preparation phase for this study occurred previously. The current study consists of the Optimization and Evaluation phases. Children aged 3-to-12 years old who are detected as "at-risk" for behavioral health disorders $(n=$ 304) at a large, urban federally qualified community health center will be referred to a Family Partner-a bicultural, bilingual member of the community with training in behavioral health and systems navigation - who will perform FN. Families will then be randomized to one of 16 possible combinations of FN delivery strategies $(2 \times 2 \times 2 \times 2$ factorial design). The primary outcome measure will be achieving a family-centered goal related to behavioral health services within 90 days of randomization. Implementation data on the fidelity, acceptability, feasibility, and cost of each strategy will also be collected. Results from the primary and secondary outcomes will be reviewed by our team of stakeholders to optimize FN delivery for implementation and dissemination based on effectiveness, efficiency, and cost.
\end{abstract}

Discussion: In this protocol paper, we describe how the MOST framework can be used to improve intervention delivery. These methods will be useful for future studies testing intervention delivery strategies and their impact on implementation.

Trial registration: ClinicalTrials.gov, NCT03569449. Registered on 26 June 2018.

Keywords: Family Navigation, Multiphase Optimization Strategy, Child behavioral health services, Health disparities

\footnotetext{
* Correspondence: sarabeth.broder-fingert@bmc.org

${ }^{1}$ Boston Medical Center, 801 Albany Street, Boston, MA 02114, USA

${ }^{2}$ Boston University School of Medicine, Boston, MA, USA

Full list of author information is available at the end of the article
}

(c) The Author(s). 2019 Open Access This article is distributed under the terms of the Creative Commons Attribution 4.0 International License (http://creativecommons.org/licenses/by/4.0/), which permits unrestricted use, distribution, and reproduction in any medium, provided you give appropriate credit to the original author(s) and the source, provide a link to the Creative Commons license, and indicate if changes were made. The Creative Commons Public Domain Dedication waiver (http://creativecommons.org/publicdomain/zero/1.0/) applies to the data made available in this article, unless otherwise stated. 


\section{Contributions to the literature}

This study offers the following additions to the literature:

- Traditional two-arm randomized controlled trials are limited in that they provide information about the general effectiveness of a packaged intervention. In contrast, the Multiphase Optimization Strategy (MOST) framework requires analysis of components and determines the optimized version of an intervention.

- The MOST framework is particularly relevant to scaled implementation of Family Navigation (FN) interventions because of overall concerns related to cost, efficiency, and effectiveness of this intervention across contexts.

- This study protocol offers an innovative application of the MOST framework to improve the delivery of FN, which is an intervention that aims to improve accessibility of health services to historically underserved populations.

\section{Background}

\section{Intervention optimization}

Delivery of behavioral interventions can be complex, as the majority of interventions consist of multiple components that can differ by order (e.g., simultaneously, sequentially, or both), locations (e.g., home, medical setting), method (e.g., in-person, remote), or individual (i.e., teacher, therapist). The importance of developing and testing efficient, effective delivery strategies for complex interventions has recently emerged as an important facet of intervention research [1]. Yet, few methodologies allow for both the rigor of a randomized clinical trial and the flexibility and adaptability of designing and testing delivery for scale. This mismatch (i.e., the narrow focus of a randomized trial in which one or two strategies can be compared with the need for testing multiple delivery strategies) has contributed to a catalog of interventions that are "evidence-based," yet without efficient strategies for delivery.

In 2005, Collins and colleagues developed the MOST framework [2], a guide for intervention developers that draws heavily from the fields of engineering, statistics, biostatistics, and behavioral science [3]. MOST involves three phases: Preparation, Optimization, and Evaluation. The Preparation phase consists of developing a conceptual model for the intervention; pilot testing; identifying "core components"; and determining what outcomes should be optimized (e.g., effectiveness, efficiency, cost). The Optimization phase uses a multifactorial design to conduct a randomized factorial experiment of specific components identified during the Preparation phase. Finally, the Evaluation phase consists of reviewing results of the trial and developing consensus regarding intervention components. Since its initial publication [4], multiple studies have utilized MOST to develop and test intervention components [5-10] with a primary focus on effectiveness $[4,10,11]$.

\section{Optimizing intervention delivery for scale: Family Navigation as an exemplar}

Another promising use of the MOST framework is optimizing intervention delivery. Many interventions-particularly complex interventions-have components that are fixed, but require a variety of delivery strategies for patients or clients that are efficient and effective [1]. FN is an example of a complex, evidence-based intervention. $\mathrm{FN}$ is a care management strategy designed to reduce disparities in care [12]. Traditional models utilize trained community health workers who assist families in overcoming systems and patient barriers to services over a time-limited period. FN is rooted in the chronic care model [13] and has evidence in multiple diseases as a means to reduce disparities by shortening the interval between discovery of risk (e.g., a positive screening mammogram for breast cancer) and diagnostic ascertainment [14-25].

Despite the promise of FN, studies demonstrate varying success upon translation from controlled research to real-world practice [26-30]. FN is a complex, multicomponent intervention which incorporates motivational interviewing (MI), problem-solving, education, and care coordination [29, 31]. FN can be delivered through a range of strategies: clinic-based meetings, home visits, or telehealth. FN delivery can be costly and timeconsuming [32]. Learning how to optimize FN delivery by determining which strategies are most effective and efficient is critical to scalability and sustainability. At the same time, understanding FN's cost as well as who benefits most is critical to decisions about how to optimally deploy available resources and generate the most costeffective, equitable benefit.

\section{Frameworks}

This study will rely on two frameworks, MOST and the Consolidated Framework for Implementation Research (CFIR) [33]. MOST was created as a means for developing "better interventions" by comparing components with the goal of optimization. In the current paper, we describe our protocol in which we use MOST to identify the most effective delivery package of FN. Use of this novel framework and study design offers the opportunity to optimize FN delivery, using empirical data, to support dissemination. Our primary objective is to compare how four different FN delivery strategies impact FN's ability to enhance access to behavioral health services. Strategies we will compare include (1) enhanced care coordination technology vs. usual care, (2) community/home-based delivery 
vs. clinic-based delivery, (3) intensive symptom tracking vs. usual symptom tracking, and (4) individually tailored vs. structured, schedule-based visits. Outcomes of interest are access to behavioral health services. We will estimate the impact of the four delivery strategies on this primary outcome, and perform exploratory analyses regarding interactions between delivery conditions and interactions with patient characteristics. We will also assess implementation outcomes of fidelity, feasibility, acceptability, and cost quantitatively. Then, we will conduct semi-structured qualitative interviews based on the CFIR to further explore these implementation constructs. Finally, using consensus methods, we will combine data to develop an optimized delivery strategy.

\section{Methodology/design}

\section{Overview}

We will use a randomized, multifactorial design to simultaneously test four FN delivery strategies, as well as combinations of the strategies, ranging from the most basic (core FN) to the most intensive (enhanced care coordination technology + community visits + enhanced symptom monitoring + structured schedule-based visits). We will evaluate which combinations of delivery strategies are most effective and efficient in regard to the primary outcome (accessing behavioral health services). Stakeholders will then evaluate data to develop an optimized model of FN delivery. The study received approval from the Boston University Institutional Review Board (Protocol Number H-37634; ClinicalTrials.gov, NCT0356944) (Additional files 1 and 2).

\section{Setting}

The study setting is a federally qualified community health center in a diverse, urban neighborhood in Boston, Massachusetts that serves $>3000$ children per year in the study's target age of 3-12 years old. Approximately $85 \%$ of patients are from a racial/ethnic minority group with $>80 \%$ using Medicaid. In addition to medical services, the health center provides comprehensive child behavioral health services. Multilingual social workers, licensed mental health clinicians, and a psychiatrist provide behavioral care in an onsite behavioral health department, and behavioral health clinicians integrated within primary care provide assessment and brief intervention.

\section{Participants}

We will enroll 304 children and their families to be randomized to a combination of four delivery strategies (see Fig. 1). All children seen at the study site are screened for behavioral health concerns at all well-child visits or when parents raise behavioral concerns. For children aged 3 to 5 years, the Preschool Pediatric Symptom Checklist (PPSC) [34], which is part of the Survey of Well-being of Young Children (SWYC) [35], will be used. For those aged 6 to 12years old, the Pediatric Symptom Checklist-17 (PSC-17) [36-39] will be administered. If agreeable, families of children identified with a behavioral health concern will be referred to the study.

To promote enrollment and retention of participants, we will enroll families regardless of language and provide bicultural and bilingual services in Spanish and Vietnamese.

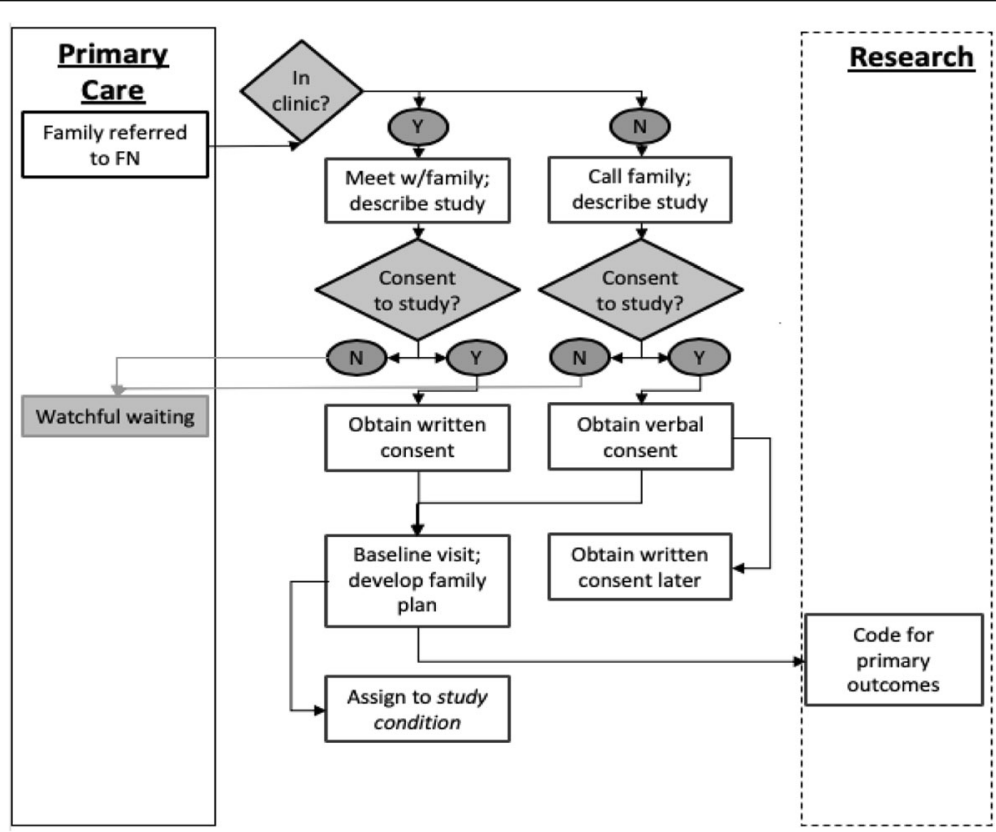

Fig. 1 Recruitment process map 
Our Navigators-in this study called Family Partners (FPs) - are bilingual in either English/Spanish or English/ Vietnamese, the most commonly spoken languages at the health center. Both the PSC-17 and SWYC are available in multiple languages. We will use telephonic translation services as needed. To promote collection of data across all study time points, FPs will be available to help families complete questionnaires in person, over the phone, or electronically. Families will receive weekly reminders when questionnaires are due.

We will also include a "watchful waiting" group for families of referred children who are not interested in accessing child behavioral services. The FP will ask parents if she can reach out again after 3 months to see if they desire services for the child at that time. Upon her recontacting the family, they will have the opportunity to enroll in the study if they would like services and wish to work with the FP.

\section{Core Family Navigation intervention}

The core components of FN [12] will be delivered by an FP, a paraprofessional community member trained to support families of children with behavioral health needs. The FN mechanisms are shown in Fig. 2. Study procedures are designed to align with existing health center workflows. Participants will be allowed to receive concomitant care of any kind while participating in the trial. All families receive the following:

1. Universal screening and behavioral health referral. FN begins with a response to a positive behavioral health screening or parent concern for behavioral health issues. The FP will provide psychoeducation and use MI to explore family preferences regarding further evaluation, and referral to behavioral health services.

2. Supporting access to behavioral health services. The FP will work with the family to access recommended services, support family preferences, and engage in treatment through the creation of a Family Plan, which includes setting family-centered goals.

3. Engagement in evidence-based treatment. FN aims to support adherence to recommendation for behavioral healthcare. The FP, who is trained in MI and collaborative decision-making, will use these skills to support parental engagement in the behavioral health treatment plan.

4. Monitoring to achieve family goals. The FP continues until the goals articulated in the Family Plan are achieved, at which point the FP will be available as needed for up to 6 months.

5. Family strengthening. FPs will refer families to local support groups and parent mental health services if needed.

6. Connection to concrete resources. FPs receive extensive training on available local resources and connect families to community-based resources (e.g., disability insurance).

\section{Family contact with FP First contact}

Initial contact with the FP will occur either in person or by phone within $48 \mathrm{~h}$ of referral. The FP will describe the study, obtain consent, administer baseline assessments, and determine a primary family-centered goal. The family will then be randomized to a study condition through a centralized randomization generator.

\section{Development of a service plan}

In consultation with the primary care team and the behavioral health clinician, the FP will work with the family to develop a plan for services, which may include onsite integrated behavioral health services, school-based services, and/or referral to an external behavioral health clinician or agency.

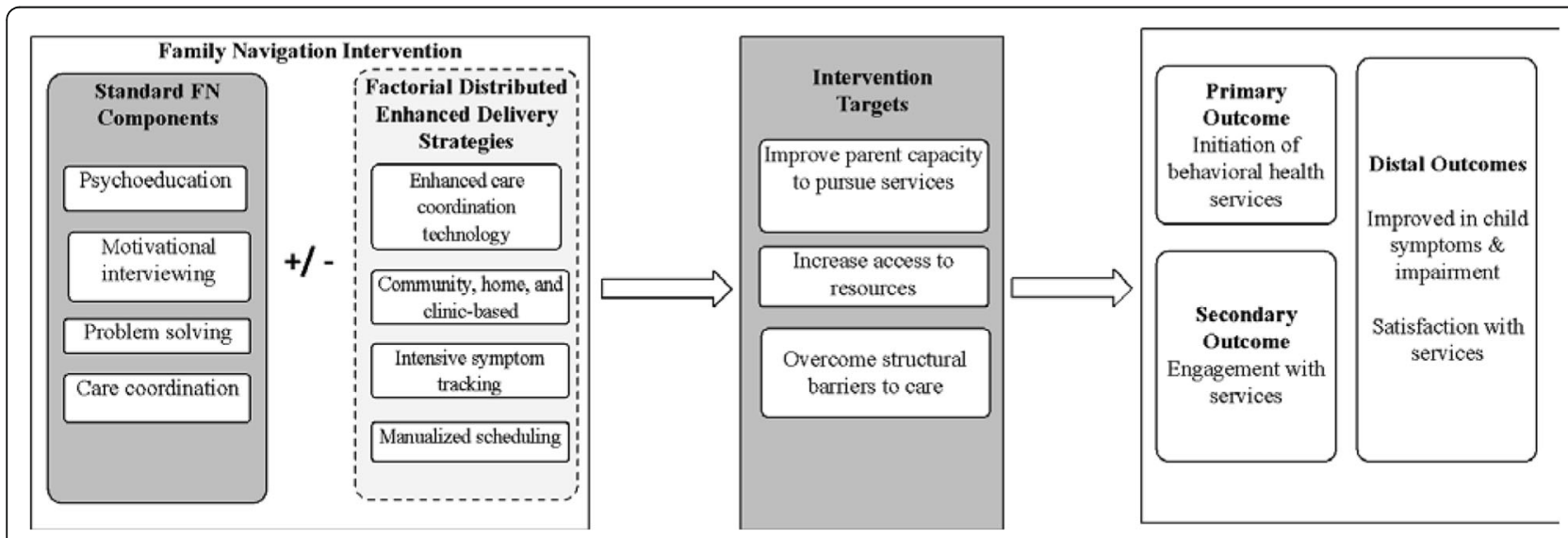

Fig. 2 Mechanisms of Family Navigation 


\section{Linkage with services}

The family will receive assistance with referral and care coordination based on the child's needs and family interest. The FP will ensure referrals are made and scheduled, and that barriers are explored through activities such as text reminders and transportation assistance.

\section{Engagement with FP}

Families' ongoing FP engagement will be guided by the core components of FN. The FP will document all activities and contacts within the electronic health record (EHR). We expect that the range of activities might include assistance in obtaining school evaluations; linkage to community-based supports such as parent groups; troubleshooting challenges to accessing services; and coordinating services between primary care, school, and specialty services.

Study conditions: Family Navigation delivery components We will test four delivery strategies using a factorial design (see Fig. 3). Families will be randomized to one of 16 combinations of delivery strategies (factors): Strategy A, Care Coordination (i.e., usual care vs. enhanced: technology assisted); Strategy B, Location (i.e., clinic-based vs. clinic + community); Strategy C, Symptom Tracking (i.e., pediatric surveillance at annual well-child visit vs. enhanced: tracking at 3, 6, 9, and 12 months); Strategy D, Visit Structure (i.e., individually tailored visits vs. structured, schedule-based visits). The specific FN delivery components are described in the following sections.. Regardless of the combination of delivery strategies (core or enhanced strategies), families will all be provided the core FN intervention.

\section{Strategy A: standard FN vs. enhanced: technology assisted} In core FN, FPs keep records and communicate with families using telephones and EHRs. In the enhanced condition, FPs will also have access to a cloud-based care coordination and communication software that offers administration of online questions, videoconferencing, and portals that can be used by parents and providers (e.g., FP, pediatrician, teacher). Families randomized to have access to care coordination software will work with the FP to become familiar with the features. The FP will introduce this technology to relevant school staff, e.g., the child's teacher.

\section{Strategy B: clinic-based vs. enhanced: clinic + community} In core FN (clinic-based), FPs are restricted to working at the clinic. Interactions will occur in person at the clinic and remotely via phone, text message, or other communication software. In the enhanced condition, FPs will be available to meet families in their homes and community, in addition to the clinic visits, and they will accompany families to community-based meetings. While out-ofclinic visits may substantially increase costs due to the FP's travel (time and mileage), we hypothesize it will also improve engagement with services.

\section{Strategy C: standard pediatric symptom surveillance vs. enhanced symptom tracking at 3, 6, 9, and 12 months}

In core FN (surveillance at well-child visits), monitoring is determined by standard pediatric practice (annually). In experimental conditions with "enhanced monitoring," FPs will monitor symptoms using validated instruments quarterly (the tools differ based on age) and communicate results to the child's care team.

\section{Strategy D: schedule-based vs. flexible meeting schedule}

In core FN, FPs provide content at their own discretion based on perceived family needs, there is no predetermined structure for meetings, and FPs may meet with families on an as-needed basis. In experimental conditions with scheduled visits, FPs will be expected to follow a curriculum that includes monthly meetings covering relevant topics.

\section{Subject allocation procedures}

Before initiation of FN, FPs will assign each family an experimental condition using a computer program. The computer program will use both a randomly generated number and "minimization procedures" to minimize imbalances across conditions with respect to target variables, including family/child characteristics (e.g., gender, race/ethnicity, language). In this procedure, the first participant is assigned at random. Subsequent participants have a $p$ chance of being randomly assigned and a $1-p$ chance of being automatically assigned to the condition that would most reduce imbalance based on selected sample characteristics. Minimization procedures are considered best practices for sequential assignment [40-43]. Randomization across four binary factors results in 16 possible combinations (see Fig. 3). We plan to enroll 304 families: $n=38$ for each cell. Unblinding of the participants will not be necessary because of the open-label nature of the trial.

\section{Outcomes}

All children will be followed for 12 months after enrollment. Measures will be collected at enrollment and at 3, 6,9 , and 12 months. The primary method of data collection is electronic parent questionnaires administered through the EHR. As needed, such as when parents are not able to read questionnaire items, FPs will be available to assist parents in their completion of the forms. For fidelity checks and outcome data collected through medical record review, we will use blinded outcome assessors. See Fig. 4 for a timetable of the study's 


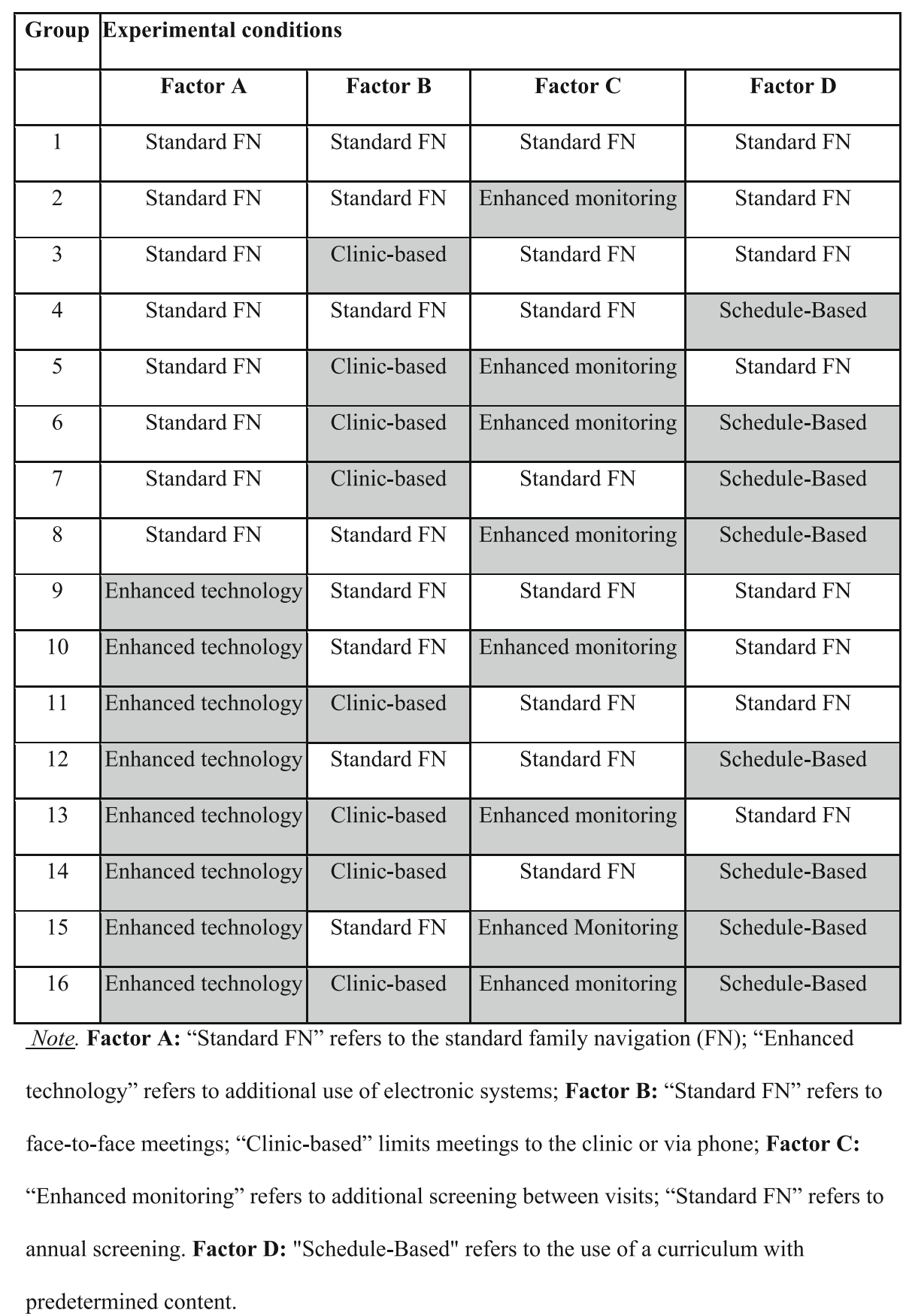

Fig. 3 Full factorial experimental design testing four delivery strategies $\left(4^{2}: 2 \times 2 \times 2 \times 2\right)$

enrollment schedule, interventions, assessments, and visits for participants.

\section{Primary measures}

The study's primary objective, access to behavioral health services, will be measured as completion of the primary family-centered behavioral health goal outlined in the Family Plan within 90 days (yes/no). For example, for families who set a goal related to engaging in behavioral health treatment, completing the primary goal will be defined as attending a behavioral health appointment within 90 days of randomization. Related to this primary objective, we will evaluate time to receipt of behavioral health services, defined as time from randomization to receipt of primary behavioral health service. Dates will be obtained from administrative and billing data (EHR) for services within the recruitment health center site and FP documentation for services 


\begin{tabular}{|c|c|c|c|c|c|c|}
\hline & \multicolumn{6}{|c|}{ STUDY PERIOD } \\
\hline & Enrollment & Allocation & & & & \\
\hline Timepoint & $-T_{1}$ & 0 & $T_{1}$ & $T_{2}$ & $\mathbf{T}_{3}$ & $T_{4}$ \\
\hline Enrollment: & $\mathrm{X}$ & & & & & \\
\hline Eligibility Screen & $\mathrm{X}$ & & & & & \\
\hline Informed consent & $\mathrm{X}$ & & & & & \\
\hline Baseline Visit & $\mathrm{X}$ & & & & & \\
\hline Allocation & & $\mathrm{X}$ & & & & \\
\hline Interventions: & & & & & & \\
\hline Standard FN & & & 4 & $\rightarrow$ & & \\
\hline Standard FN + Enhanced monitoring & & & $\leftarrow$ & $\rightarrow$ & & \\
\hline Standard FN + Clinic-based & & & 4 & $\rightarrow$ & & \\
\hline Standard FN + Curriculum-based & & & 4 & $\rightarrow$ & & \\
\hline Standard FN + Clinic-based + Enhanced monitoring & & & $\leftarrow$ & $\rightarrow$ & & \\
\hline $\begin{array}{l}\text { Standard FN + Clinic-based + Enhanced monitoring + } \\
\text { Curriculum-based }\end{array}$ & & & 4 & $\rightarrow$ & & \\
\hline Standard FN + Clinic-based + Curriculum-based & & & 4 & $\rightarrow$ & & \\
\hline Standard FN + Enhanced monitoring + Curriculum-based & & & $\leftarrow$ & $\rightarrow$ & & \\
\hline Enhanced Technology + Standard FN & & & 4 & $\rightarrow$ & & \\
\hline $\begin{array}{l}\text { Enhanced Technology + Standard FN + Enhanced } \\
\text { Monitoring }\end{array}$ & & & 4 & $\rightarrow$ & & \\
\hline Enhanced Technology + Clinic-based + Standard FN & & & 4 & $\rightarrow$ & & \\
\hline Enhanced Technology + Standard FN + Curriculum-based & & & $\leftarrow$ & $\rightarrow$ & & \\
\hline $\begin{array}{l}\text { Enhanced Technology + Clinic-based + Enhanced } \\
\text { Monitoring + Standard FN }\end{array}$ & & & $\leftarrow$ & $\rightarrow$ & & \\
\hline $\begin{array}{l}\text { Enhanced Technology }+ \text { Clinic-based }+ \text { Standard FN }+ \\
\text { Curriculum-based }\end{array}$ & & & $\leftarrow$ & $\rightarrow$ & & \\
\hline $\begin{array}{l}\text { Enhanced Technology + Standard FN + Enhanced } \\
\text { Monitoring + Curriculum-based }\end{array}$ & & & 4 & $\rightarrow$ & & \\
\hline $\begin{array}{l}\text { Enhanced Technology + Clinic-based + Enhanced } \\
\text { Monitoring + Curriculum-based }\end{array}$ & & & 4 & $\rightarrow$ & & \\
\hline Assessments: & & & & & & \\
\hline Demographics & $\mathrm{X}$ & & & & & \\
\hline Pediatric Symptom Checklist-17 & $\mathrm{X}$ & & $\mathrm{X}$ & $\mathrm{X}$ & $\mathrm{X}$ & $\mathrm{X}$ \\
\hline The Survey of Wellbeing of Young Children (SWYC) & $\mathrm{X}$ & & $\mathrm{X}$ & $\mathrm{X}$ & $\mathrm{X}$ & $\mathrm{X}$ \\
\hline Client Satisfaction Questionnaire 8 (CSQ-8) & & & & $\mathrm{X}$ & & \\
\hline Interpersonal Relationship with Navigator (PSN-I) & & & & $\mathrm{X}$ & & \\
\hline $\begin{array}{l}\text { The Parental Attitudes Towards Psychological Services } \\
\text { Inventory (PATPSI) }\end{array}$ & $\mathrm{X}$ & & & $\mathrm{X}$ & & $\mathrm{X}$ \\
\hline Family Resource Scale (FRS) & $\mathrm{X}$ & & & $\mathrm{X}$ & & $\mathrm{X}$ \\
\hline
\end{tabular}

Fig. 4 Study timetable

outside the health center. Additional engagement and child-level outcomes are described in the following paragraphs. See Table 1 for details on each outcome defined across five elements including domain, specific measurement, metric, method of aggregation, and time point.

Engagement in care will be defined as $\geq 4$ visits with a behavioral health provider within 90 days of first FP visit, or resolution of service need as determined by behavioral health provider [44].

Child functioning will be measured depending on the child's age. For children aged 6years and older, the
Pediatric Symptom Checklist-17 (PSC-17) will be used as a broad measure of child functioning. The PSC-17 is a 17item psychosocial screen designed to recognize cognitive, emotional, and behavioral problems. Three subscales, Internalizing, Attention, and Externalizing, have specific cutoffs and provide additional guidance regarding need for further follow-up. The PSC-17 is embedded in the Epic (EHR) as a self-scoring form. It is widely used and has been validated in diverse populations [36-39]. For children under $5 \frac{1}{2}$ years of age, the Survey of Well-being of Young Children (SWYC) will be used to measure child 
Table 1 Outcome definitions across five elements

\begin{tabular}{|c|c|c|c|c|}
\hline Domain & Specific measurement & Specific metric & $\begin{array}{l}\text { Method of } \\
\text { aggregation }\end{array}$ & Time points \\
\hline $\begin{array}{l}\text { Health services } \\
\text { utilization }\end{array}$ & $\begin{array}{l}\text { EHR record review of encounter for behavioral } \\
\text { health service need }\end{array}$ & $\begin{array}{l}\text { Time to event: total number of days between } \\
\text { randomization and first service encounter related } \\
\text { to behavioral health need }\end{array}$ & $\begin{array}{l}\text { Proportion } \\
\text { with an } \\
\text { event }\end{array}$ & $\begin{array}{l}\text { Continuous } \\
\text { days from } \\
-T_{1} \text { to } T_{2}\end{array}$ \\
\hline $\begin{array}{l}\text { Engagement in } \\
\text { behavioral } \\
\text { healthcare }\end{array}$ & $\begin{array}{l}\text { Documentation of FP visit dates and EHR } \\
\text { record review of behavioral health service } \\
\text { appointment dates and provider } \\
\text { recommendations }\end{array}$ & $\begin{array}{l}\geq 4 \text { visits with a behavioral health provider within } \\
90 \text { days of first FP visit, or resolution of service need } \\
\text { as determined by behavioral health provider }\end{array}$ & Mean & $\mathrm{T}_{1}$ \\
\hline $\begin{array}{l}\text { Child functioning } \\
\text { (children aged } 5.5^{\text {years and older) }}\end{array}$ & Pediatric Symptom Checklist-17 & Total score summed from 17 items & $\begin{array}{l}\text { Proportion } \\
\text { above } \\
\text { clinical } \\
\text { cutoff }\end{array}$ & $-\mathrm{T}_{1}, \mathrm{~T}_{4}$ \\
\hline $\begin{array}{l}\text { Child functioning } \\
\text { (children under }^{\mathrm{a}} \\
5.5 \text { years of age) }\end{array}$ & $\begin{array}{l}\text { The Survey of Well-being of Young Children } \\
\text { (SWYC) Preschool Pediatric Symptom Checklist }\end{array}$ & Total score summed from 18 items & $\begin{array}{l}\text { Proportion } \\
\text { above } \\
\text { clinical } \\
\text { cutoff }\end{array}$ & $-\mathrm{T}_{1}, \mathrm{~T}_{4}$ \\
\hline $\begin{array}{l}\text { Satisfaction with } \\
\text { health services }\end{array}$ & Client Satisfaction Questionnaire 8 (CSQ-8) & Total score summed from 8 items & Mean & $\mathrm{T}_{2}$ \\
\hline $\begin{array}{l}\text { Interpersonal } \\
\text { relationship with } \\
\text { Family Partner }\end{array}$ & Interpersonal Relationship with Navigator (PSN-I) & Total score summed from 9 items & Mean & $\mathrm{T}_{2}$ \\
\hline Parent attitudes & $\begin{array}{l}\text { Parental Attitudes Toward Psychological } \\
\text { Services Inventory (PATPSI) }\end{array}$ & Total score summed from 21 items & Mean & $-\mathrm{T}_{1}, \mathrm{~T}_{2}, \mathrm{~T}_{4}$ \\
\hline $\begin{array}{l}\text { Parental mental } \\
\text { health }^{\mathrm{a}}\end{array}$ & Patient Health Questionnaire-2 (PHQ-2) & Total score summed from 2 items & $\begin{array}{l}\text { Proportion } \\
\text { above } \\
\text { clinical } \\
\text { cutoff }\end{array}$ & $-T_{1}, T_{4}$ \\
\hline $\begin{array}{l}\text { Access to } \\
\text { community } \\
\text { resources }\end{array}$ & Family Resource Scale (FRS) & Total score summed from 30 items & Mean & $-T_{1}, T_{2}, T_{4}$ \\
\hline Structural barriers & $\begin{array}{l}\text { Tool for Health and Resilience in Vulnerable } \\
\text { Environments (THRIVE) survey }\end{array}$ & $\begin{array}{l}\text { Total number of social needs reported across } 8 \\
\text { possible areas }\end{array}$ & Mean & $-T_{1}, T_{2}, T_{4}$ \\
\hline Service use & EHR record review & $\begin{array}{l}\text { Among scheduled visits, percentage of visits that } \\
\text { were completed (i.e., show rate) }\end{array}$ & $\begin{array}{l}\text { Mean } \\
\text { percentage }\end{array}$ & $\begin{array}{l}\text { Monthly } \\
\text { reports }\end{array}$ \\
\hline
\end{tabular}

$-T_{1}$ enrollment, $T_{1} 3$ months post enrollment, $T_{2} 6$ months post enrollment, $T_{3} 9$ months post enrollment, $T_{4} 12$ months post -enrollment

${ }^{a}$ Families randomized to the enhanced symptom tracking condition will receive this measure at $-T_{1}, T_{1}, T_{2}, T_{3}$, and $T_{4}$

functioning. The SWYC [35] screens for cognitive, motor, language, and social-emotional development among children up to $5 \frac{1}{2}$ years of age. We will track symptoms using the SWYC's Preschool Pediatric Symptom Checklist (PPSC) [34], an 18-item questionnaire that has demonstrated strong validity and acceptability in diverse populations. Translations are available in a range of languages.

\section{Secondary measures}

Secondary patient experience outcomes will be measured at baseline and at 3, 6, 9, and/or 12 months. Secondary outcome measures will be used to evaluate the theory-based mechanisms of FN effectiveness and indicate for whom the FN delivery strategy is most effective (i.e., assessment of mediators and moderators). Theory-based mechanisms are both related to the person (e.g., attitudes) and the system (i.e., access to resources, overcome barriers). See Table 2 for the specific intervention targets and theoretical foundations.
Satisfaction with health services will be with measured with the Client Satisfaction Questionnaire 8 (CSQ-8); i.e., it will be used to assess family satisfaction with health services. This measure has established psychometric properties with ethnically diverse populations [45-47].

Interpersonal relationship with FP will be measured using the Interpersonal Relationship with Navigator (PSN-I). This measure is a validated 9-item scale with strong psychometric properties in samples of culturally diverse, underserved patients [48].

To assess parent attitudes, we will use the Parental Attitudes Toward Psychological Services Inventory (PATPSI). This measure consists of 21 Likert-type items ( $0=$ strongly disagree; $5=$ strongly agree $)$ that assess parents' help-seeking attitudes, help-seeking intentions, and mental health stigma [49].

To assess parental mental health, the Patient Health Questionnaire-2 (PHQ-2) [50,51] will be administered. 
Table 2 Theory-based mechanisms and measures

\begin{tabular}{llll}
\hline Domain & Target & Theoretical mechanism & Instrument \\
\hline Person & Parent experience & $\begin{array}{l}\text { Improved parental attitudes about mental health increases } \\
\text { capacity to engage in services }\end{array}$ & PATPSI \\
& Parent experience & Improved mental health increases capacity to engage in services & Patient Health Questionnaire-2 \\
System & Access to resources & Improving social determinants increases access to resources & Family Resource Scale \\
& Structural barriers & Coordination decreases structural barriers & EHR FP templates \\
\hline
\end{tabular}

The PHQ-2 is a validated two-question depression screener with score ranges from 0 to 6 . The authors of the measure identified a score of 3 as the optimal cut point when using the PHQ-2 to screen for depression. If the score is 3 or greater, major depressive disorder is likely.

Access to community resources of families will be measured with the Family Resource Scale (FRS) [52, 53], a 30 -item scale that assesses family concerns regarding adequacy of resources. We hypothesize that FN will improve access to resources over time. The FRS assesses numerous family needs and factors including growth/ support, health/necessities, physical necessities, physical shelter, intra-family support, communication/employment, child care, and personal resources. It is a selfadministered instrument; each item is rated on a 5-point scale ranging from "not at all adequate" to "almost always adequate."

Structural barriers will be alleviated with the collection of monthly professional contact data, using FN logs on contacts with others on the care team. We will measure level of care coordination (number and frequency of contacts between care providers) and existence of barriers as delineated in the logs. Additionally, Tool for Health and Resilience in Vulnerable Environments (THRIVE) data will be collected as part of usual care and recorded in the EHR [54]. THRIVE is a social determinant of health survey based on parent-reported need across eight domains: housing, food, utilities, paying for medications, caregiving, transportation, payment for medical employment, and education.

Data regarding service use will be collected through the EHR. This will include the FP's documentation and show rate (i.e., appointments scheduled and completed).

\section{Implementation measures}

For each delivery strategy, we will collect data on fidelity, acceptability, feasibility, and cost.

Fidelity to the FN core model and delivery strategies We will use multiple data sources to assess fidelity using methods employed in prior studies [16-19, 25-27, 29]. We will review structured navigation visit templates integrated into the EHR and FP contact logs monthly. A random sample of two visits per month will be reviewed using a Navigation Checklist we developed for "realtime" monitoring. MI will be assessed quarterly using audiotaped standardized patient interactions, which will be scored using the Motivational Interviewing Supervision and Training Scale (MISTS) $[55,56]$. We will assess both fidelity to the core model and fidelity to the delivery strategies; for example, we will check if FPs are administering symptom monitoring, and how frequently, in the "symptom tracking" condition. If an FP is not meeting fidelity criteria, she will be provided with retraining and additional support. Once per year, the FPs will participate in an MI booster training session to maintain their MI skills.

Acceptability and feasibility We will use qualitative methods to assess acceptability of each delivery strategy. We will use purposeful sampling to interview five subjects from each of the 16 strategies $(n=80)$. Interview questions will be based on the CFIR. Because the goal of the study is delivery optimization, we will specifically focus our questions on the eight domains that comprise the "Intervention Characteristics" construct within the CFIR: source, evidence strength and quality, relative advantage, adaptability, trialability, complexity, design quality and packaging, and cost.

Cost We will use time-driven activity-based costing $(T D A B C)$ to develop cost estimates for each delivery strategy [57]. TDABC's goal is to develop valid estimates of service costs while minimizing expenditures on research [57]. It accomplishes this goal by requiring only two key sets of estimates: capacity cost rate and demand for resource capacity. Because the explicit goal of using TDABC in our project is to support process optimization and enhance scalability, we will not calculate costs over the entire care delivery value chain [58]. Instead, we focus only on specific FN activities, consistent with the use of TDABC in studies of healthcare processes [59].

We will first calculate the capacity cost rate for the FP as a function of total annual compensation divided by the time available for FN activities. Next, we will estimate the demand for resource capacity resulting from each FN delivery strategy. Assumptions regarding FN activities that create demand for resource capacity will be 
based on process maps [59]. Process maps developed during a previous study of FN will be adapted and refined to reflect FN procedures in the current project [60]. Based on these process maps, staff reports, and direct observations, we will develop time equations for each FN delivery strategy. Such time equations closely resemble linear regression equations in that they include an intercept that reflects a baseline time estimate, as well as coefficients and dummy variables that reflect additional time required to account for variations. For example, a time equation for an FN phone call to a patient might include an intercept of 2 min to look up a phone number and make a call, a coefficient that adds $5 \mathrm{~min}$ if the patient answers, and an additional $10 \mathrm{~min}$ if a survey is administered.

\section{Data management}

All participants will be assigned a unique study code. This study code will be used to link data from the EHR, billing records, and Act.MD. The crosswalk that links study codes to participant names will be kept in a locked office and separate from the data. This crosswalk will be kept for the duration of the study and then destroyed. Any paper surveys utilized will be transferred the same day that they are completed to a locked file cabinet in the locked office of a study Principal Investigator.

All collected data will be stored on a Health Insurance Portability and Accountability Act (HIPAA)-compliant, password-protected, secure drive maintained by the institutions sponsoring the study. The study utilizes data collection and management tools that meet HIPAA security rules to protect the confidentiality and security of protected health information.

\section{Statistical analyses Overview}

All statistical analyses will be done in SAS (v9.4) and MPlus (v8). Baseline characteristics of parents and children will be compared across conditions to assess balanced randomization. Characteristics include race/ ethnicity, insurance status, primary language, and child characteristics (e.g., age).

\section{Main effects}

Following an intent-to-treat model, multiple regression models will be used to test hypotheses regarding the main effects of the four delivery strategies and their combined effects on the study's primary outcome. A series of increasingly complex models will be constructed to address each specific index of outcome. For example, a logistic regression analysis will test receipt of behavioral health services within 90 days. Cox regression (proportional hazards) analyses will then be used to test the effect of each factor on time to receipt of services.
Similarly, engagement in services will first be analyzed with logistic regression using our definition of engagement in care as a binary outcome, with subsequent multilevel models to analyze engagement in multiple services.

We will examine all data for missing information and loss to follow-up. We plan to consult with a statistician regarding any missing data and will use multiple imputation as appropriate. For outcomes involving engagement, sensitivity analyses will be conducted in which missing data from the EHR is interpreted as failure to engage in services. While we do not hypothesize interactions among the delivery strategies, these will also be explored. Following recommendations for factorial designs, effect coding (not dummy coding) will be used for experimental conditions to assess for interaction. In addition to evaluating effects "at the margins" using all available cells, results for each individual cell will also be reported [61], as will simple main effects.

\section{Mediator/moderator analyses to examine intervention mechanism}

Consistent with our theoretical model and based on our prior studies and literature review, we hypothesize that FN intervention effects will be mediated by parents' capacity to pursue services, access to services, and structural barriers. We will examine mediational effects using two different, but related, methods: the approach of Baron and Kenny and the use of path analysis models. Each approach can be used to differentiate between direct and indirect intervention effects. In the path analysis models (which have greater statistical power), we will create a series of nested models based on our theoretical model in which we will systematically vary model parameters and constraints to test the effect of each potential mediator. Nested models will be compared using difference tests and other standard indices (Akaike's information criterion, the comparative fit index (optimal value > 0.95 ), the Tucker-Lewis index (optimal value $>0.95$ ), and the root mean square error of approximation (optimal values $<0.06)$ ). We will fit these models with MPlus software, which allows for the modeling of continuous and dichotomous, endogenous, and exogenous variables. While our study design only allows for direct testing of the causal effects of primary delivery strategies A, B, C, the causal effect of mediating variables can be analyzed by treating factors as instrumental variables in the path analysis $[62,63]$.

\section{Moderator analyses}

We will evaluate the extent to which each delivery strategy, race/ethnicity, primary language, and symptom severity moderate FN effects using stratified analysis. Previous studies have found no effect of such 
demographic variables on the effect of FN. We hypothesize that any effects will be small and clinically non-significant; however, we will perform these analyses, as evaluation of moderators is important to ensure equity.

\section{Sample size and power}

Of the three measures to operationalize engagement, we powered our study on the dichotomous variable "achieved goal related to receipt of mental health services within 90 days" (the most conservative estimate). We based our power calculation on the number needed to detect the smallest differences in primary outcomes that are of clinical importance. Our formative work with staff at the recruitment health center and other community health centers indicated that a relative risk of approximately $25 \%$ would be considered clinically significant. Therefore, if $60 \%$ of families in the core FN condition engage in mental health services (estimates based on our prior work), in order to detect a $25 \%$ difference (i.e., $75 \%$ of families in any of the FN delivery conditions engage in services) and assuming two-tailed tests and a type 1 error rate of $5 \%$, approximately 304 participants are required to detect this effect ( $n=19$ in each of 16 cells).

We expect strong effects of study mediators, in particular fidelity variables and variables that are central to our theoretical model, such as increased parent capacity. We estimate that our design will have at least $80 \%$ power to detect mediation effects where the paths from independent variable to mediator and from mediator to outcome are of at least small-to-medium Effect Size $(E S=.26)$. Given that our mediation analyses are designed to support decisions regarding intermediate outcomes to be tracked for quality control and assurance, effect sizes less than this magnitude are not considered to be clinically important. In contrast, analyses of patient-level treatment moderators are exploratory, as we have no evidence to support hypotheses of any effect.

\section{Qualitative data analysis}

Each interview will be independently coded by two members of the research team, using the CFIR codebook. The interviews will then be collectively reviewed to ensure coding consensus and reconcile discrepancies. A review of all of the codes for each interview will be conducted until members of the research team reach consensus as to which codes should be applied to specific segments of text. After consensus is achieved among coders, interview transcripts will then be entered, coded, and analyzed in QSR-NVivo.

\section{Final evaluation}

The final stage of the MOST framework-Evaluationwill be conducted after completing data analysis. We will convene key stakeholders in behavioral health, FN, and policy to develop consensus recommendations regarding FN delivery. Data on effectiveness of each component both in isolation and in combination, secondary outcomes, and implementation will be presented to our stakeholder panel. Then, a modified Delphi approach [64] will be used to select components for inclusion in the final intervention package.

\section{Post-trial care}

Access to FN through the study will end with the termination of the trial. However, participants will be able to continue accessing care within and outside of the federally qualified community health center, whether or not the FP played a role in supporting access to that care. In the absence of FPs available to support families through study participation, families will follow general clinical protocols for behavioral health case management and referral.

\section{Discussion}

In the current study we use the MOST framework to optimize FN delivery. Using a factorial design, we will develop an optimized, efficient, effective version of FN. The goal of the intervention is to improve access to, and engagement in, diagnostic and treatment services for children with behavioral health disorders. This approach represents important advances in the field of implementation science for several reasons.

First, we are using the MOST framework to optimize intervention delivery. While MOST is a framework for optimization, it is important to note that what one optimizes on (e.g., clinical outcomes, implementation, cost) is determined by the key stakeholders involved in the study. Thus, in this study we are specifically using MOST as a framework for delivery. This study protocol can serve as a guide for others working to optimize intervention delivery. The framework holds a unique benefit in that the majority of methodologies used for optimization do not include the rigor of randomization. Thus, understanding these methods can be of great value to the field.

Second, these methods can help inform others looking to optimize an intervention designed to alleviate disparities in access to services. Low-income and ethnically diverse children with mental/behavioral health concerns often experience delays in obtaining a diagnosis and appropriate evidence-based treatments $[65,66]$. Solutions to mitigate these disparities, such as FN, must be delivered in an efficient and effective manner to increase the likelihood of sustainable, widespread adoption. Furthermore, FN has been implemented in real-world practice with different strategies, and with varying success [26-28]. The current study will advance our understanding of which delivery 
strategies are the most effective, and for whom. Given that some delivery strategies are more labor- and resourceintensive than others, to be efficient, FN programs understand "active ingredients" that lead to the most positive outcomes while leaving out potentially expensive and time-consuming strategies with lesser impact.

Finally, current health service delivery reforms are promoting primary care networks as the "hub" of care coordination. Financial incentives created under the Affordable Care Act are spawning new systems that link primary care and specialty services within integrated networks. For example, Accountable Care Organizations (ACOs) [67] are groups of doctors, hospitals, and other healthcare providers who join together to give coordinated, high-quality care. Implementation of FN within the setting of a newly formed ACO (Boston Accountable Care Organization) links this innovation to the broader policy context and maximizes scale-up potential within emerging delivery systems. Understanding delivery within this new context is important to FN's ultimate success.

\section{Challenges and potential solutions}

The two main challenges we anticipate are related to the complexity of subject assignment and the pragmatic nature of the study. Specifically, because we are using the MOST framework and a factorial design, we will be randomizing families to one of 16 conditions. There is therefore a large burden on the study team to prevent contamination across conditions, and monitoring for fidelity of each condition. Contamination is a universal concern for studies using the MOST framework [68]. We have implemented several strategies to prevent contamination. First, we are using an electronic randomization protocol that allows the Navigators to directly randomize to a condition in real time. This allows Navigators to know, at the time of enrollment, which of the 16 conditions a family is randomized to. This program also allows us to input which condition the family is assigned into the medical record. Keeping this data within the medical record helps ensure that whenever a FP is working with the family, she is immediately alerted as to which condition the family is assigned. Finally, each condition is given a "code name" to support accurate categorization of each family into their correct condition. This code name is documented in the medical record as well.

The second challenge relates to the fact that this is a pragmatic trial embedded within usual care of a large, federally qualified health center, and we may lose the ability to tightly control the use of each condition. For example, if a Navigator or families choose not to use the web-based care coordination software, we will not have the ability to ensure use. Although this may make assessment of outcome data difficult (i.e., effectiveness of a condition that is insufficiently used), it does allow us to better understand how conditions may be used in the "real world."

\section{Trial status}

The study start date was June 24, 2019. The current protocol version date is June 26, 2019. The protocol contributors were Emily Feinberg (Co-Principal Investigator), Lisa Fortuna (Co-Principal Investigator), Sarabeth Broder-Fingert (Co-Investigator), Radley Christopher Sheldrick (Co-Investigator), Megan Jordan (Co-Investigator), Dana Rubin (Co-Investigator), and Andrea Chu (Project Manager).

\section{Conclusions}

This study uses the MOST framework to optimize FN delivery. We are comparing a specific delivery package against the aggregate effect of all the other delivery strategies. This framework offers a more efficient strategy for testing multiple delivery strategies over a traditional multiarm trial. These methods will be useful for future investigators working to optimize interventions for implementation and dissemination.

\section{Supplementary information}

Supplementary information accompanies this paper at https://doi.org/10. 1186/s13063-019-3853-y.

Additional file 1. World Health Organization Trial Registration Data Set. Additional file 2. SPIRIT 2013 checklist: recommended items to address in a clinical trial protocol and related documents.

\section{Abbreviations}

ACO: Accountable Care Organization; ADHD: Attention deficit/hyperactivity disorder; ASD: Autism spectrum disorder; EHR: Electronic health record: FN: Family Navigation; FP: Family Partner; FRS: Family Resource Scale; MI: Motivational interviewing; MISTS: Motivational Interviewing Supervision and Training Scale; MOST: Multiphase Optimization Strategy; PHQ-2: Patient Health Questionnaire-2; PPSC: Preschool Pediatric Symptom Checklist; PSC17: Pediatric Symptom Checklist-17; PSN-I: Interpersonal Relationship with Navigator; SWYC: Survey of Well-being of Young Children (SWYC)

\section{Trial sponsors}

The sponsors are Boston Medical Center, 750 E. Harrison Ave., Boston, MA 02118 , and

the National Institute of Mental Health, 6001 Executive Boulevard, Bethesda, MD 20892.

\section{Trial oversight}

Because this project includes a clinical intervention, Family Navigation, a vulnerable population, and a modified request for waiver of consent, the inclusion of a Data and Safety Monitoring Plan and Board is appropriate. The Data and Safety Monitoring Board (DSMB) for this study includes an independent group of experts on child behavioral health, clinical trials, and biostatistics, who will advise and monitor the project with the study Principal Investigators. None of the members will directly supenvise, report to, or directly collaborate with the Principal Investigators. The DSMB will meet every 6 months. Its primary responsibilities will be to (1) review and evaluate the accumulated study data for participant safety, study conduct and progress, and efficacy; and (2) make recommendations concerning the continuation, modification, or termination of the trial. In this project the DSMB will consider study-specific data and any concerns for poor clinical outcomes among patients in a particular 
randomization condition. Any changes to the study protocol will be submitted to the institution's Institutional Review Board (IRB) for approval, reported to the ClinicalTrials.gov registry, shared with the $\mathrm{NIMH}$ project officer, and discussed by the DSMB. Handling of biological specimens is not applicable to this trial, as none will be collected for any outcomes.

\section{Adverse events and harms}

There are no anticipated harms from this study. However, because the research covers the topic of mental health and potential psychosocial stressors, participation may be emotionally distressing to individuals in the study. All staff will be fully trained by the Principal Investigators and FN supervisor on how to work with children and families, including working with the families of children with behavioral healthcare needs, as well as mandated reporting procedures and management of emergency situations including suicidal or homicidal ideation. Families have the right to decline participation in the study and continue their other clinical services at their primary care site. For this study, the most important adverse events to monitor are breach of confidentiality and psychiatric hospitalizations, which have the potential to increase through participation in the study. However, this may be a result of improved screening, identification, and engagement in clinical services. We will track and report adverse events according to IRB regulations.

\section{Dissemination policy}

The investigators will disseminate the findings of this study through peerreviewed conference presentations, presentations to study stakeholders such as clinicians within the study setting, and through publication in peerreviewed journals. The investigators will not use professional writers. Decisions around authorship on publications will adhere to the International Committee of Medical Journal Editors (ICMJE) authorship guidelines (1).

\section{Authors' contributions}

SBF conceptualized and designed the study, designed interview questions, drafted the initial manuscript, critically reviewed the manuscript, and approved the final manuscript as submitted. JK assisted with study design, critically reviewed the manuscript, and approved the final manuscript as submitted. RCS assisted with study design, critically reviewed the manuscript, and approved the final manuscript as submitted. AC assisted with study design, critically reviewed the manuscript, and approved the final manuscript as submitted. LF assisted with study design, critically reviewed the manuscript, and approved the final manuscript as submitted. MJ assisted with study design, critically reviewed the manuscript, and approved the final manuscript as submitted. DR assisted with study design, critically reviewed the manuscript, and approved the final manuscript as submitted. EF assisted with study design, critically reviewed the manuscript, and approved the final manuscript as submitted.

\section{Funding}

This study was supported by the NIMH, grant number: R01MH11712301. The $\mathrm{NIMH}$ had no role in the study design, data collection, data management, data monitoring, data analysis, or interpretation of data. The NIMH did not contribute toward the writing of this report or the decision to submit this report for publication. The NIHM will not have ultimate authority over any of these study activities.

\section{Availability of data and materials}

The study investigators will have access to and manage the final study dataset. Data collected as part of this study will be submitted to the National Database for Clinical Trials Related to Mental IIness (NDCT) per the datasharing guidelines of the National Institute of Mental Health (NIMH) (NOT$\mathrm{MH}-14-015)$ to be shared with other investigators.

\section{Ethics approval and consent to participate}

This study received approval from the Boston University Institutional Review Board (IRB Protocol Number H-37634; ClinicalTrials.gov, NCT0356944).

\section{Consent for publication}

Not applicable.

\section{Competing interests}

The authors declare that they have no competing interests.

\section{Author details}

${ }^{1}$ Boston Medical Center, 801 Albany Street, Boston, MA 02114, USA. ${ }^{2}$ Boston University School of Medicine, Boston, MA, USA. ${ }^{3}$ Boston University School of Public Health, Boston, MA, USA. ${ }^{4}$ DotHouse Health Center, Dorchester, MA, USA.

Received: 17 August 2019 Accepted: 25 October 2019

Published online: 16 December 2019

\section{References}

1. Chambers DA, Norton WE. The Adaptome: advancing the science of intervention adaptation. Am J Prev Med. 2016;51(4 Suppl 2):S124-31.

2. Collins LM, Murphy SA, Nair VN, Strecher VJ. A strategy for optimizing and evaluating behavioral interventions. Ann Behav Med Publ Soc Behav Med. 2005;30(1):65-73.

3. Collins LM. Optimization of behavioral, biobehavioral, and biomedical interventions: the Multiphase Optimization Strategy (MOST). (Statistics for Social and Behavioral Sciences). [Internet]. New York: Springer International Publishing; 2018. https://www.springer.com/us/book/9783319722054. Accessed 19 Jun 2019

4. Wyrick DL, Rulison KL, Fearnow-Kenney M, Milroy JJ, Collins LM. Moving beyond the treatment package approach to developing behavioral interventions: addressing questions that arose during an application of the Multiphase Optimization Strategy (MOST). Transl Behav Med. 2014; 4(3):252-9.

5. Gabovitch E, Lauer E, Dutra C. Healthy People 2020 Roadmap Report for Massachusetts Children and Youth with ASD/DD [Internet]. Boston: University of Massachusetts Medical School, E.K. Shriver Center; 2016. https://shriver.umassmed.edu/research/population-health/healthy-people-2 020-roadmap. Accessed 5 Mar 2019

6. Windsor LC, Benoit E, Smith D, Pinto RM, Kugler KC, Newark Community Collaborative Board (NCCB). Optimizing a community-engaged multi-level group intervention to reduce substance use: an application of the multiphase optimization strategy. Trials. 2018;19(1):255.

7. Bernstein SL, Dziura J, Weiss J, Miller T, Vickerman KA, Grau LE, et al. Tobacco dependence treatment in the emergency department: a randomized trial using the Multiphase Optimization Strategy. Contemp Clin Trials. 2018;66:1-8.

8. Gwadz MV, Collins LM, Cleland CM, Leonard NR, Wilton L, Gandhi M, et al. Using the multiphase optimization strategy (MOST) to optimize an HIV care continuum intervention for vulnerable populations: a study protocol. BMC Public Health. 2017;17(1):383.

9. Kugler KC, Balantekin KN, Birch LL, Savage JS. Application of the multiphase optimization strategy to a pilot study: an empirical example targeting obesity among children of low-income mothers. BMC Public Health. 2016; 16(1):1181.

10. Pellegrini CA, Hoffman SA, Collins LM, Spring B. Optimization of remotely delivered intensive lifestyle treatment for obesity using the Multiphase Optimization Strategy: Opt-IN study protocol. Contemp Clin Trials. 2014; 38(2):251-9.

11. McClure JB, Derry H, Riggs KR, Westbrook EW, St JJ, Shortreed SM, et al. Questions about quitting (Q2): design and methods of a Multiphase Optimization Strategy (MOST) randomized screening experiment for an online, motivational smoking cessation intervention. Contemp Clin Trials. 2012;33(5):1094-102.

12. Broder Fingert S, Stadnick N, Hickey E, Diaz Linhart Y, Goupil J, Feinberg E. Short report: defining the core components of family navigation for autism spectrum disorder. Autism. In press.

13. Glasgow RE, Orleans CT, Wagner EH. Does the chronic care model serve also as a template for improving prevention? Milbank Q. 2001; 79(4):579-612 iv-v.

14. Marshall JK, Mbah OM, Ford JG, Phelan-Emrick D, Ahmed S, Bone L, et al. Effect of patient navigation on breast cancer screening among African American Medicare beneficiaries: a randomized controlled trial. J Gen Intern Med. 2016;31(1):68-76.

15. McKenney KM, Martinez NG, Yee LM. Patient navigation across the spectrum of women's health care in the United States. Am J Obstet Gynecol. 2018;218(3):280-6.

16. Ali-Faisal SF, Colella TJF, Medina-Jaudes N, Benz SL. The effectiveness of patient navigation to improve healthcare utilization outcomes: a meta- 
analysis of randomized controlled trials. Patient Educ Couns. 2017;100(3): 436-48.

17. Diaz-Linhart $Y$, Silverstein M, Grote N, Cadena L, Feinberg E, Ruth BJ, et al. Patient navigation for mothers with depression who have children in Head Start: a pilot study. Soc Work Public Health. 2016;31(6):504-10.

18. Guevara JP, Rothman B, Brooks E, Gerdes M, McMillon-Jones F, Yun K. Patient navigation to facilitate early intervention referral completion among poor urban children. Fam Syst Health J Collab Fam Healthc. 2016;34(3):281-6.

19. Levinson AH, Valverde P, Garrett K, Kimminau M, Burns EK, Albright $K$, et al. Community-based navigators for tobacco cessation treatment: a proof-ofconcept pilot study among low-income smokers. BMC Public Health. 2015;15:627.

20. Silverstein M, Diaz-Linhart Y, Cabral H, Beardslee W, Hegel M, Haile W, et al. Efficacy of a maternal depression prevention strategy in Head Start: a randomized clinical trial. JAMA Psychiatry. 2017;74(8):781-9.

21. Silverstein M, Hironaka LK, Walter HJ, Feinberg E, Sandler J, Pellicer M, et al. Collaborative care for children with ADHD symptoms: a randomized comparative effectiveness trial. Pediatrics. 2015;135(4):e858-67.

22. Silverstein M, Diaz-Linhart Y, Grote N, Cadena L, Cabral H, Feinberg E. Harnessing the capacity of Head Start to engage mothers with depression in treatment. J Health Care Poor Underserved. 2017;28(1):14-23.

23. Perrin EC, Sheldrick RC, MCMenamy JM, Henson BS, Carter AS. Improving parenting skills for families of young children in pediatric settings: a randomized clinical trial. JAMA Pediatr. 2014;168(1):16-24

24. Levy SL, Hill E, Mattern K, McKay K, Sheldrick RC, Perrin EC. Colocated mental health/developmental care. Clin Pediatr (Phila). 2017;56(11):1023-31.

25. Feinberg E, Abufhele M, Sandler J, Augustyn M, Cabral H, Chen N, et al. Reducing disparities in timely autism diagnosis through family navigation: results from a randomized pilot trial. Psychiatr Serv Wash DC. 2016;67(8):912-5.

26. Donelan K, Mailhot JR, Dutwin D, Barnicle K, Oo SA, Hobrecker K, et al. Patient perspectives of clinical care and patient navigation in follow-up of abnormal mammography. J Gen Intern Med. 2011;26(2):116-22.

27. Hedlund N, Risendal BC, Pauls H, Valverde PA, Whitley E, Esparza A, et al. Dissemination of patient navigation programs across the United States. J Public Health Manag Pract JPHMP. 2014;20(4):E15-24.

28. Ramachandran A, Freund KM, Bak SM, Heeren TC, Chen CA, Battaglia TA. Multiple barriers delay care among women with abnormal cancer screening despite patient navigation. J Womens Health. 2015;24(1):30-6.

29. Krok-Schoen JL, Oliveri JM, Paskett ED. Cancer care delivery and women's health: the role of patient navigation. Front Oncol. 2016;6:2 https://www. ncbi.nlm.nih.gov/pmc/articles/PMC4729879/. Accessed 29 Nov 2018.

30. Broder-Fingert S, Qin S, Goupil J, Rosenberg J, Augustyn M, Blum N, et al. A mixed-methods process evaluation of Family Navigation implementation for autism spectrum disorder. Autism Int J Res Pract. 2018;8:1362361318808460.

31. Algorta GP, MacPherson HA, Youngstrom EA, Belt CC, Arnold LE, Frazier TW, et al. Parenting stress among caregivers of children with bipolar spectrum disorders. J Clin Child Adolesc Psychol. 2018;47(SUP1):S306-20.

32. Li Y, Carlson E, Villarreal R, Meraz L, Pagán JA. Cost-effectiveness of a patient navigation program to improve cervical cancer screening. Am J Manag Care. 2017;23(7):429-34.

33. Damschroder LJ, Aron DC, Keith RE, Kirsh SR, Alexander JA, Lowery JC. Fostering implementation of health services research findings into practice: a consolidated framework for advancing implementation science. Implement Sci. 2009;4(1):50.

34. Sheldrick RC, Henson BS, Merchant S, Neger EN, Murphy JM, Perrin EC. The Preschool Pediatric Symptom Checklist (PPSC): development and initial validation of a new social/emotional screening instrument. Acad Pediatr. 2012;12(5):456-67.

35. Perrin EC, Sheldrick CR, Visco Z, Mattern K. The Survey of Well-Being of Young Children (SWYC): user's manual. Boston: Tufts Medical Center; 2016. www.theSWYC.org.

36. Murphy JM, Reede J, Jellinek MS, Bishop SJ. Screening for psychosocial dysfunction in inner-city children: further validation of the Pediatric Symptom checklist. J Am Acad Child Adolesc Psychiatry. 1992;31(6):1105-11.

37. Murphy JM, Ichinose C, Hicks RC, Kingdon D, Crist-Whitzel J, Jordan P, et al. Utility of the Pediatric Symptom Checklist as a psychosocial screen to meet the federal Early and Periodic Screening, Diagnosis, and Treatment (EPSDT) standards: a pilot study. J Pediatr. 1996;129(6):864-9.

38. Jellinek MS, Murphy JM, Robinson J, Feins A, Lamb S, Fenton T. Pediatric Symptom Checklist: screening school-age children for psychosocial dysfunction. J Pediatr. 1988;112(2):201-9.
39. Jellinek MS, Bishop SJ, Murphy JM, Biederman J, Rosenbaum JF. Screening for dysfunction in the children of outpatients at a psychopharmacology clinic. Am J Psychiatry. 1991;148(8):1031-6.

40. Taves DR. Minimization: a new method of assigning patients to treatment and control groups. Clin Pharmacol Ther. 1974;15(5):443-53.

41. Scott NW, MCPherson GC, Ramsay CR, Campbell MK. The method of minimization for allocation to clinical trials. a review. Control Clin Trials. 2002;23(6):662-74.

42. Pocock SJ, Simon R. Sequential treatment assignment with balancing for prognostic factors in the controlled clinical trial. Biometrics. 1975; 31(1):103-15.

43. Higham $R$, Tharmanathan P, Birks $Y$. Use and reporting of restricted randomization: a review. J Eval Clin Pract. 2015;21(6):1205-11.

44. Olfson M, Mojtabai R, Sampson NA, Hwang I, Druss B, Wang PS, et al. Dropout from outpatient mental health care in the United States. Psychiatr Serv Wash DC. 2009;60(7):898-907.

45. Larsen DL, Attkisson CC, Hargreaves WA, Nguyen TD. Assessment of client/ patient satisfaction: development of a general scale. Eval Program Plann. 1979;2(3):197-207.

46. Roberts RE, Atrkisson CC, Mendias RM. Assessing the Client Satisfaction Questionnaire in English and Spanish. Hisp J Behav Sci. 1984;6(4):385-96.

47. Kelly PJ, Kyngdon F, Ingram I, Deane FP, Baker AL, Osborne BA. The Client Satisfaction Questionnaire-8: psychometric properties in a cross-sectional survey of people attending residential substance abuse treatment. Drug Alcohol Rev. 2018;37(1):79-86.

48. Jean-Pierre P, Fiscella K, Winters PC, Post D, Wells KJ, McKoy JM, et al. Psychometric development and reliability analysis of a patient satisfaction with interpersonal relationship with navigator measure: a multi-site patient navigation research program study. Psychooncology. 2012;21(9):986-92.

49. Turner EA. The parental attitudes toward psychological services inventory: adaptation and development of an attitude scale. Community Ment Health J. 2012;48(4):436-49.

50. Kroenke K, Spitzer RL, Williams JBW. The Patient Health Questionnaire-2: validity of a two-item depression screener. Med Care. 2003;41(11):1284-92.

51. Kroenke K, Spitzer RL, Williams JBW, Löwe B. The Patient Health Questionnaire Somatic, Anxiety, and Depressive Symptom Scales: a systematic review. Gen Hosp Psychiatry. 2010;32(4):345-59.

52. Brannan AM, Manteuffel B, Holden EW, Heflinger CA. Use of the family resource scale in children's mental health: reliability and validity among economically diverse samples. Admin Pol Ment Health. 2006;33(2):182-97.

53. Dunst CJ, Leet HE. Measuring the adequacy of resources in households with young children. Child Care Health Dev. 1987;13(2):111-25.

54. THRIVE. Tool for health and resilience in vulnerable environments: final project report executive summary [Internet]. Oakland: Prevention Institute; 2004. https://www.preventioninstitute.org/sites/default/files/uploads/ THRIVE\%20overview\%20and\%20background.pdf

55. Madson MB, Campbell TC, Barrett DE, Brondino MJ, Melchert TP. Development of the Motivational Interviewing Supervision and Training Scale. Psychol Addict Behav J Soc Psychol Addict Behav. 2005;19(3):303-10.

56. Madson MB, Campbell TC. Measures of fidelity in motivational enhancement: a systematic review. J Subst Abus Treat. 2006;31(1):67-73.

57. Kaplan RS. Anderson SR. Time-driven activity-based costing: a simpler and more powerful path to higher profits; 2007. https://www.hbs.edu/faculty/ Pages/item.aspx?num=23236. Accessed 26 Jun 2019

58. Kaplan RS, Porter ME. How to solve the cost crisis in health care. Harv Bus Rev. 2011;89(9):46-52, 54, 56-61 passim.

59. Keel G, Savage C, Rafiq M, Mazzocato P. Time-driven activity-based costing in health care: a systematic review of the literature. Health Policy Amst Neth. 2017;121(7):755-63.

60. Broder-Fingert S, Qin S, Goupil J, Rosenberg J, Augustyn M, Blum N, et al. A mixed-methods process evaluation of Family Navigation implementation for autism spectrum disorder. Autism Int J Res Pract. 2019;23(5):1288-99.

61. McAlister FA, Straus SE, Sackett DL, Altman DG. Analysis and reporting of factorial trials: a systematic review. JAMA. 2003;289(19):2545-53.

62. Shrout PE, Bolger N. Mediation in experimental and nonexperimental studies: new procedures and recommendations. Psychol Methods. 2002; 7(4):422-45.

63. Mackinnon DP, Fairchild AJ, Fritz MS. Mediation analysis. Annu Rev Psychol. 2007;58:593-614.

64. Dalkey NC. The Delphi method [Internet]. 1969. https://www.rand.org/pubs/ research_memoranda/RM5888.html. Accessed 21 Jun 2019. 
65. McGuire TG, Miranda J. New evidence regarding racial and ethnic disparities in mental health: policy implications. Health Aff Proj Hope. 2008;27(2):393-403.

66. Lu W. Child and adolescent mental disorders and health care disparities: results from the National Survey of Children's Health, 2011-2012. J Health Care Poor Underserved. 2017;28(3):988-1011.

67. Newman D. CRS report for Congress: Accountable Care Organizations and the Medicare Shared Savings Program. Washington, DC: Congressional Research Service; 2011. p. 1-46.

68. Guastaferro K, Collins LM. Achieving the goals of translational science in public health intervention research: the Multiphase Optimization Strategy (MOST). Am J Public Health. 2019;109(S2):S128-9.

\section{Publisher's Note}

Springer Nature remains neutral with regard to jurisdictional claims in published maps and institutional affiliations.

Ready to submit your research? Choose BMC and benefit from:

- fast, convenient online submission

- thorough peer review by experienced researchers in your field

- rapid publication on acceptance

- support for research data, including large and complex data types

- gold Open Access which fosters wider collaboration and increased citations

- maximum visibility for your research: over $100 \mathrm{M}$ website views per year

At BMC, research is always in progress.

Learn more biomedcentral.com/submissions 